\title{
Instandhaltungsbenchmarking mit Fokus Stahlindustrie
}

\author{
Hubert Biedermann und Robin Kühnast \\ Lehrstuhl Wirtschafts- und Betriebswissenschaften, Leoben, Österreich \\ Eingegangen 6. November 2018; angenommen 24. November 2018; online publiziert 10. Januar 2019
}

Zusammenfassung: Steigende Verfügbarkeits- und Zuverlässigkeitsanforderungen in Kombination mit den Markterfolgsfaktoren Agilität, Qualität und Kosten verlangen von anlagenintensiven Industrien nach einer strategischen Ausgestaltung des Asset Managements. In diesem Kontext gewinnt die Instandhaltung nicht nur zunehmend strategische Bedeutung, sondern kann sich auch bei entsprechend ausgestalteten Managementinstrumentarien als Wertschöpfungstreiber etablieren. Zur Einschätzung, in welchem Reifegrad sich abgegrenzte Technologie- und Anlagenbereiche der Stahlindustrie mit ihren Instandhaltungsorganisationen befinden, wurde eine umfassende Benchmarkstudie im deutschsprachigen Raum durchgeführt, deren Ergebnisse hier auszugsweise dargestellt werden.

Schlüsselwörter: Instandhaltungsmanagement, Benchmarking, Managementinstrumentarium, Stahlindustrie

Maintenance Benchmarking with a Focus on the Steel Industry

Abstract: Increasing availability and reliability requirements - in combination with the market success factors agility, quality, and costs-require strategic asset management. Therefore maintenance is gaining an increasing strategic importance and can establish a value creation driver with appropriately designed management tools. A comprehensive benchmark study was carried out in German-speaking countries to assess the maturity of the technology and plant areas of the steel industry with their maintenance organisations. The results of this study are presented here in parts.

Keywords: Maintenance management, Benchmarking, Controlling instruments, Steel industry

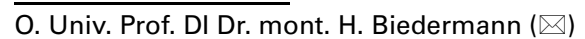
Lehrstuhl Wirtschafts- und Betriebswissenschaften, Franz Josef Straße 18,

8700 Leoben, Österreich

hubert.biedermann@unileoben.ac.at

\section{Einleitung und Grundbegriffe}

Benchmarking stellt ein Analyse- und Orientierungsinstrument dar, das durch kontinuierlichen Vergleich von Produkten, Dienstleistungen, Prozessen, Instrumenten und Methoden mit (mehreren) Unternehmen oder Organisationseinheiten die Leistungslücken systematisch identifiziert.

Instandhaltungsbenchmarking wird hier verstanden als permanenter Prozess im Konzept der schlanken, wissens- und lernbasierten Instandhaltung (lean smart maintenance) zur Identifikation von Best Practice Lösungen als Basis zur Leistungsverbesserung durch kontinuierlichen externen Vergleich. Dieser fokussiert auf die Strategie, die Organisation und den Prozess der Leistungserstellung im Rahmen des Instandhaltungsmanagements und schließt die Organisationskultur mit ein [1].

Man unterscheidet internes, wettbewerbsorieniertes und funktionales Benchmarking. Die vorliegende Studie stellt ein funktionales branchenübergreifendes Benchmarking dar, wobei die Anwendung eines ausdifferenzierten Managementsystems und die zugrundeliegenden Instandhaltungskonzepte vergleichbar bleiben. Da diese Methodik bewusst die deutlichen Unterschiede im Leistungsprofil der Instandhaltung verschiedener Branchen nutzt, bietet diese eine gute Ausgangsbasis für innovative Ansätze. Die Einführung eines effektiven und zielgerichteten Benchmarkings als Instrument zur Optimierung betrieblicher Leistungen, Abläufe und Prozesse wird zumeist in Kombination mit Reifegradmodellen für ein innovatives, lernendes Instandhaltungsmanagement zunehmend selbstverständlich. Mit steigender Anlagenintensität, wechselnden Auslastungssituationen, zunehmender Anlagennutzung (teilkontinuierlich bis kontinuierlich), steigender Anlagenverkettung (Prozessindustrie) steigt ganz allgemein der Instandhaltungsbedarf. Im quantitativen Kennzahlen- und Methodenvergleich müssen sich diese Branchen- und Unternehmenstypologien am höchsten Reifegrad orientieren. Zwischen den Fähigkeiten des besten Unternehmens der jeweiligen Branche und dem theoretischen Optimum liegen oftmals noch beträchtliche Potenziale, die es im eigenen Unternehmen auszuschöpfen gilt [1]. 
Abb. 1: Branchenverteilung der Benchmarkstudie [2]
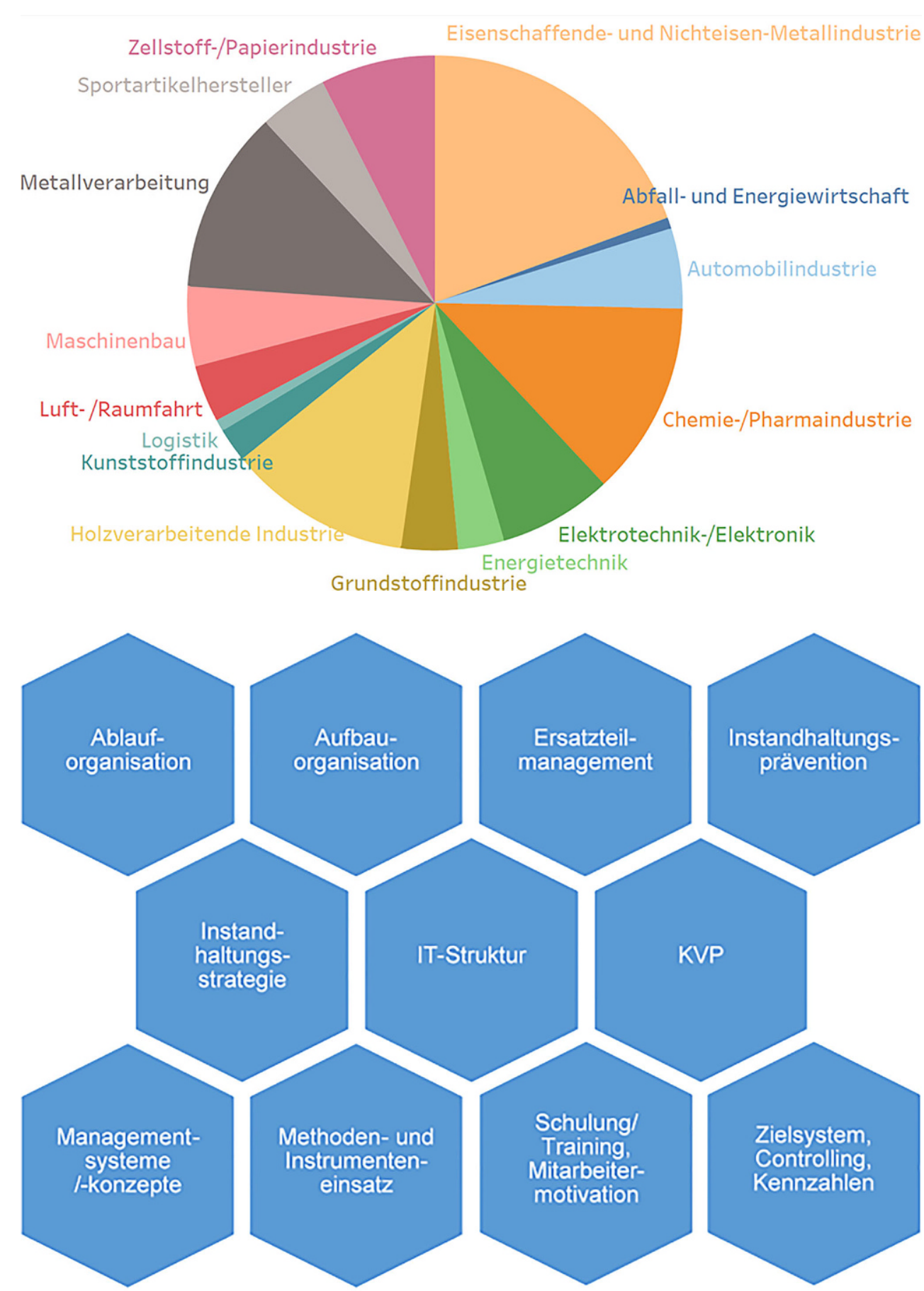

Abb. 2: Bewertungskategorien der Benchmarkstudie [2]

Gleichzeitig darf nicht übersehen werden, dass bei fehlerhafter Anwendung des Benchmarkings Gefahren in dieser Methodik ruhen. Der Gefahr "Paralyse durch Analyse" durch die Erhebung immer weiterer unüberschaubarer (scheingenauer) Daten sollte vermieden werden.

\section{Motivation und Ziel der Studie}

Die vorliegende Studie gibt einen branchenübergreifenden Überblick über den Reifegrad der Instandhaltung ausge- wählter Betriebsstandorte in 11 Managementkategorien. Dabei wird aufgezeigt, in welchen Managementinstrumenten Instandhaltungsorganisationen der DACH-Region noch Potenziale aufweisen und welche Standards als Best Practice gelten. Als Zielgruppe der vorliegenden Studie ist die Führungsebene adressiert; diese soll einen Einblick in das Managementinstrumentarium der Instandhaltung und zudem Hinweise auf Potenziale zur Verbesserung der eigenen Organisation erhalten. 
Abb. 3: Reifegradverteilung der in der Studie berücksichtigten Firmen unter Hervorhebung der Stahlindustrie (Durchschnitt und 26 Betriebsstandorte in Österreich und Deutschland)

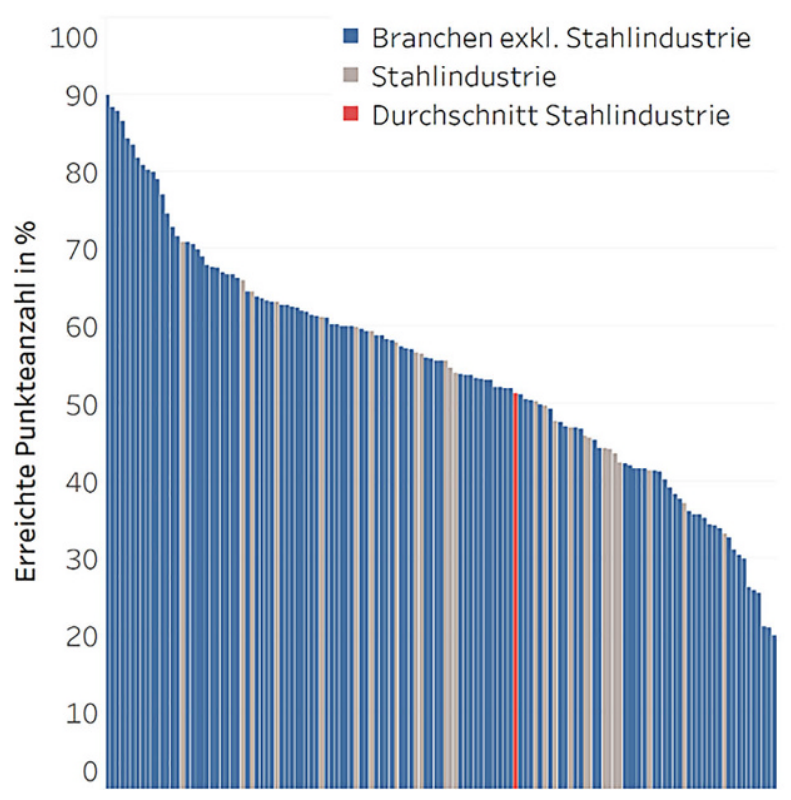

\subsection{Umfang der Studie und Branchenzuordnung der berücksichtigten Firmen}

Lean Smart Maintenance verfolgt die wertschöpfungsorientierte Gestaltung des Instandhaltungsmanagements als Grundlage für eine effiziente Instandhaltung. Diese Wertschöpfungsorientierung ist in den letzten Jahren durch die steigende Digitalisierung und Systemkomplexität für Industriebetriebe immer wichtiger geworden.

In dieser Studie sind 134 Instandhaltungen (standortbezogene Instandhaltungsbetriebe von Betrieben und Firmen) berücksichtigt. So vielseitig die einzelnen Unternehmen und deren Instandhaltungsmanagement aufgebaut sind, so sind es die an der Befragung teilnehmenden Branchen. Die für die Generierung der Benchmarks benötigten Unternehmensdaten stammen neben der jährlichen Vergabe des Maintenance Award Austria aus der laufenden Projektarbeit mit Industriepartnern durch den Lehrstuhl für Wirtschafts- und Betriebswissenschaften der Montanuniversität Leoben. Aufgrund der weitreichenden Netzwerke der ÖVIA und des Lehrstuhls konnten im Laufe der Jahre bereits 200 Fragebögen evaluiert werden.

Wie in Abb. 1 ersichtlich, sind die vier am häufigsten teilgenommenen Branchen dieser Auswertung [2]:

- Eisenschaffende- und Nichteisen-Metallindustrie (19,1\% aller ausgewerteten Fragebögen; knapp 1/4 aller Fragebögen)

- Chemie und Pharmaindustrie $(12,5 \%)$

- Metallverarbeitungsindustrie (12,5\%)

- Holzverarbeitende Industrie (11,7\%)

Des Weiteren sind ebenfalls die Automobilindustrie, die Elektrotechnik \& Elektronik sowie die Zellstoff und Papierindustrie mit einem großen Anteil an den Umfragen beteiligt.

Die Datenerhebung erfolgt durch einen Fragebogen. Diesem ist ein Reifgradmodell zugrunde gelegt, welches in
11 Kategorien gegliedert ist. Zur Plausibilitätsprüfung erfolgt in ausgewählten Betrieben ein site visit. Die Auswertung der Angaben erfolgt mit einem instandhaltungsspezifischen Bewertungsschlüssel. Die Datenbank ermöglicht, anonymisierte Benchmarkdaten interessierten Unternehmen zur Verfügung zu stellen. Darüber hinaus können neben der Weiterentwicklung der Instandhaltungsorganisationen in den letzten Jahren auch branchenspezifische Potenziale aufgezeigt werden.

Die Bewertungskategorien sind in Abb. 2 dargestellt.

Jeder der Bewertungskategorien ist eine bestimmte Punkteanzahl zugeordnet, welche wiederum auf die einzelnen Fragen aufgeteilt ist. Die Gewichtung der Antworten reicht von 0 bis 5 Punkten je nach Reifegrad. Die Punkte werden sowohl innerhalb der einzelnen Managementinstrumente als auch über die Kategorien sowie im Gesamtfragebogen summiert. Im Anschluss erfolgt der Vergleich mit der höchsten zu erreichbaren Punkteanzahl und dem brancheninternen Best-Practice-Wert über alle Ebenen. Dadurch können die Stärken und Schwächen der Instandhaltungsorganisation ermittelt und Verbesserungsmaßnahmen abgeleitet werden. Mit höchstem Reifegrad kann eine Instandhaltungsorganisation 55 Punkte (100\%) erreichen.

\subsection{Ausgewählte Ergebnisse für die Stahlindustrie}

Im Zeitraum von 2015 bis 2018 wurden 26 Betriebsstandorte bzw. Technologiebereiche mit ihren Instandhaltungsorganisationen untersucht. Diese Technologiebereiche umfassen Kokerei, Sinteranlage, Hochofen, Stahlwerk, weiterverarbeitende Betriebe einschließlich Gießwalzanlagen bis hin zu Feinstblecherzeugung und dem innerbetrieblichen Transport (Eisenbahnsysteme). 


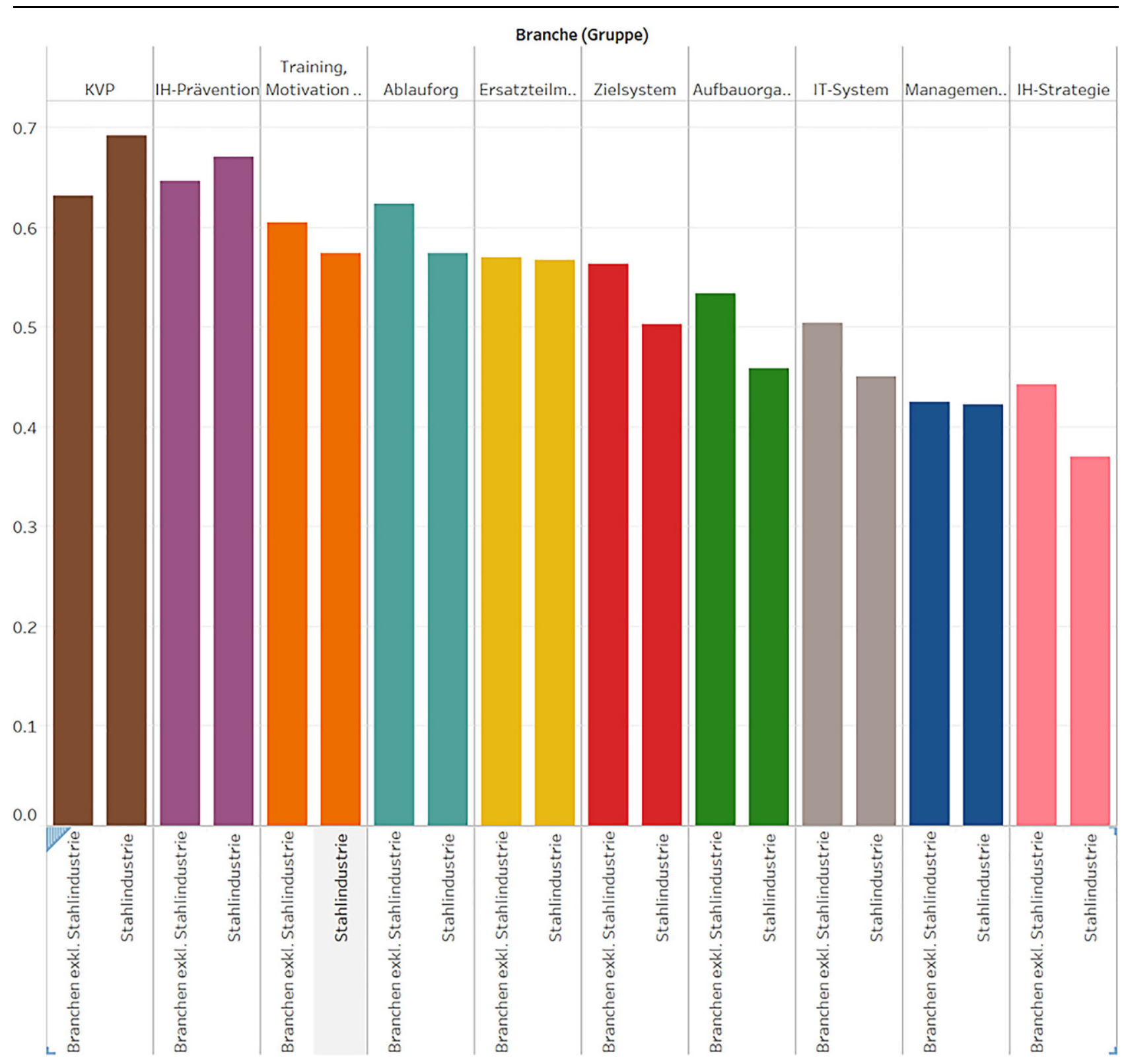

Abb. 4: Reifegrad ausgewählter Managementkategorien im Vergleich Stahlindustrie zu Gesamtindustrie

Abb. 3 zeigt das Spektrum der Reifegrade aller berücksichtigten 134 Instandhaltungsorganisationen.

- $7 \%$ sind der Kategorie "Best Practice"

- $31 \%$ der Kategorie "Gut"

- $46 \%$ der Kategorie "Durchschnitt“ und

- $16 \%$ der Kategorie "Unterdurchschnittlich“

\section{zuzuordnen.}

Damit wird deutlich, dass mehr als die Hälfte (über $60 \%$ ) noch beträchtliche Potenziale vorfinden, die eine Verbesserung des Instandhaltungsmanagements ermöglichen. Da davon auszugehen ist, dass dieses Potenzial die Verfügbarkeit der betreuten Anlagen und die Instandhaltungskosten verbessern, bieten sich für zahlreiche Firmen hohe wertsteigernde ökonomische Vorteile.
Für die Stahlindustrie wird sichtbar, dass sich die Instandhaltungsorganisationen großteils (19 von 26) im Feld des durchschnittlichen Reifegrades befinden; lediglich fünf haben gutes Niveau und keine Organisation ist in der Klasse Best Practice.

Werden ausgewählte Kategorien des Managementinstrumentariums ausgewählt und der Vergleich mit den restlichen Branchen (siehe Abb. 1) vorgenommen, ist zu erkennen, dass Potenziale in der Organisation, dem Zielund Controllingsystem, der adäquaten IT-Unterstützung und der Gestaltung und Dynamisierung der Instandhaltungsstrategie bestehen (Abb. 4). 
Am Beispiel der Managementkategorie "Instandhaltungsstrategie“ sind die Defizite insbesondere in der fehlenden

- normativen Orientierung durch ein Instandhaltungsleitbild mit Zielintegration

- objektorientierten Ableitung des Strategiemix

- Flexibilisierung der Wartungs- und Inspektionsintervalle

- Bewertung autonomer Tätigkeiten

- risikoorientierten Lagerung der Ersatzteile

- analytisch begründeten Outsourcingstrategie

- bzw. unzulänglich ausgestalteten IT-Unterstützung zur risikobasierten Budgetierung und Schwachstellenanalyse

- Integration in ein Lean Management und weiterer Managementsysteme

- horizontal und vertikal durchgängigen Zielorientierung in Kombination mit Visible Management

zu finden.

Stärken finden sich in der prozessorientierten Ablauforganisation, der Implementierung von Sicherheits- und Energiemanagementsystemen, der Organisation von Revisionen und Großstillständen sowie der Ersatzteilbewirtschaftung.

\section{Resümee}

Das Aufgabenfeld der Instandhaltung ist breit gefächert und verlangt Kompetenzen aus technischen, betriebswirtschaftlich-managementorientierten und sozialen Bereichen. In einem kompetitiven Markt mit den Megatrends Digitalisierung und Globalisierung, die zusätzlichen Druck ausüben, reicht es nicht mehr, die klassischen Kompetenzfelder, wie funktionales und strukturelles Technikwissen, zu beherrschen und das klassische Führungsinstrumentarium Planung und Organisation anzuwenden. Es gilt eine ganzheitliche Sicht auf das Aufgaben- und Arbeitsspektrum im Sinne des life-cycle orientierten Asset-Managements in der Instandhaltung zu etablieren und eine hohe Reife in möglichst vielen Teilbereichen des Anlagenmanagements zu erlangen. Digitale Methoden werden die Aufgabendurchführung und hier insbesondere die Inspektionen unterstützen und in geeigneten Fällen eine prädiktive Instandhaltung zulassen. Darüber hinaus wird Kreativität sowie das Extrahieren von Informationen aus komplexen Prozessen im Zuge der Schwachstellenanalyse zunehmend an Bedeutung gewinnen [3].

Die Positionierung von Produktionsbereichen in der Wertschöpfungskette bis hin zum Halbfertig- und Fertigprodukt der Stahlindustrie zeigt im funktionalen Benchmarking zu vergleichbaren Branchen (hohe Anlagen-, Material- und Energieintensität) teilweise erhebliche Potenziale auf. Es gilt, möglichst rasch diese Potenziale zu heben und Konzepte wie Lean-Smart-Maintenance (Instandhaltung 4.0) zu etablieren [4].

Funding. Open access funding provided by Montanuniversität Leoben.

Open Access Dieser Artikel wird unter der Creative Commons Namensnennung 4.0 International Lizenz (http://creativecommons.org/licenses/ by/4.0/deed.de) veröffentlicht, welche die Nutzung, Vervielfältigung, Bearbeitung, Verbreitung und Wiedergabe in jeglichem Medium und Format erlaubt, sofern Sie den/die ursprünglichen Autor(en) und die Quelle ordnungsgemäß nennen, einen Link zur Creative Commons Lizenz beifügen und angeben, ob Änderungen vorgenommen wurden.

\section{Literatur}

1. Biedermann, H.: Benchmarking - Chancen und Risiken für die Instandhaltung, in Biedermann, H. (Hrsg.): Benchmarking - Auf dem Weg zu Best Practice in Produktion und Instandhaltung, Köln: TÜVVerlag, 1998, S. 27-45

2. Passath, Th.; Biedermann, H.: Bewertungskategorien und Studienergebnisse, in Biedermann, H. (Hrsg.): Benchmark - Instandhaltung, Köln: TÜV Media, 2018, S.19ff.

3. Biedermann, H.; Kühnast, R.: Ergebnisse der Benchmarkstudie, in Biedermann, H. (Hrsg.): Benchmark - Instandhaltung. Köln: TÜV Media, 2018, S.139

4. Biedermann, H.: Lean Smart Maintenance - wertschöpfende, lernorientierte und ressourceneffiziente Instandhaltung, in Biedermann, H. (Hrsg.): Lean Smart Maintenance - Konzepte, Instrumente und Anwendungen für eine effiziente und intelligente Instandhaltung, KöIn: TÜV Media, 2016, S. 19-29 